\section{Testimonio y cicatriz de la ciudad \\ Mecanismos para el \\ Santiago neoliberal del \\ Estado de Excepción de 1973}

FRANCISCO CARDEMIL PÉREZ*
CLASIFICACIÓN Investigación

TEMA Historia y territorio

DESARROLLO $\mathbf{2}^{\text {do }}$ semestre 2016

INSTITUCIÓN Pontificia Universidad Católica de Chile

PROFESOR/A Alejandra Celedón

CONTACTO facardemil@uc.cl

\section{RESUMEN}

El problema de acceso a una vivienda en Chile tuvo una de sus etapas más complejas en la segunda mitad del siglo xx. Durante este tiempo se llevaron a cabo las Operaciones Confraternidad, las primeras respuestas de vivienda social de la dictadura, cuya implementación y ejecución fue cuestionada y resentida de acuerdo a la prensa de oposición de la época. Estas operaciones consistieron principalmente en el traslado de personas y fueron aplicadas en una gran cantidad de comunas en el Gran Santiago. Su objetivo declarado era eliminar la extrema pobreza, convirtiendo a la población en propietarios.

La ejecución de estas operaciones no pareció ser siempre igual. Este estudio pone a prueba tres mecanismos que darían cuenta de aquellas estrategias neoliberales dentro de las Operaciones Confraternidad. Ellos corresponden a la excepción legal, es decir, la supresión de las leyes en un territorio determinado para lograr ciertos fines; la especulación del mercado de suelos y la geopolítica pinochetista que consiste en una visión del desarrollo urbano que entiende a la ciudad como un organismo que se autorregula y que crece hacia las periferias. A través del objeto como testimonio del movimiento de la pobreza se intentará esclarecer estos mecanismos y las relaciones que los unen y comunican en los objetivos ideológicos detrás de los traslados de las operaciones, aquellos objetivos paralelos a la erradicación de la pobreza.

Palabras Clave Erradicación de la pobreza, Traslado, Objeto, Excepción legal, Operaciones Confraternidad.

\section{ABSTRACT}

The social housing issue in Chile had one of its most complex phases in the second half of the $20^{\text {th }}$ century. During this period, the Operaciones Confraternidad were implemented, as a first response to social housing during the dictatorship, their execution and implementation both were questioned and resented, according the opposition press at the time. These operations mainly consisted of uprooting people and were applied in many comunas over the Grand Santiago area. The declared objective was the eradication of extreme poverty, turning the common population into homeowners.

The execution of these Operations seemed to differ from case to case. This study tests three of the mechanisms that could identify the underlying neoliberal strategies of the Operaciones Confraternidad. These mechanisms consist of: legal exceptions, meaning, the suppresion of law in certain territories to achieve specific goals; real estate speculation; and, finally, the Pinochetist geopolitics, which refers to an urban development view that understands the city as a self-regulating organism that grows towards its peripheral areas. Through the object as a means of accounting for the uprooting of the poor, this study will clarify these mechanisms and the similarities that link and communicate ideological objectives, the true objectives behind the eradication of poverty.

KEYWORDS Poverty eradication, Uprooting, object, Legal exceptions, Operaciones Confraternidad. 


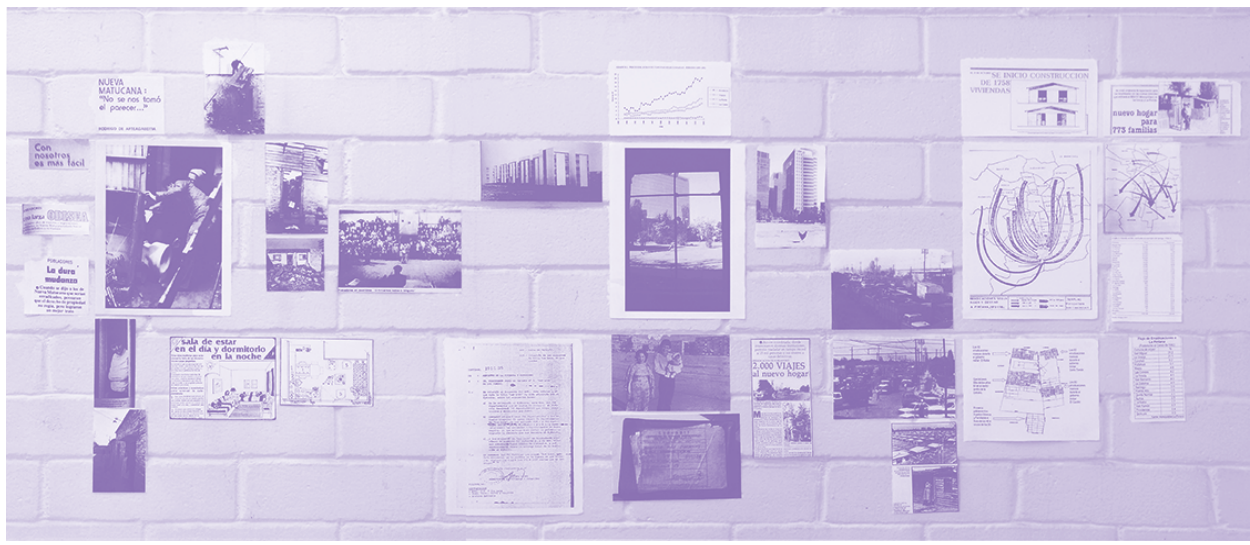

FIGURA 1.

Mapa registral de documentos sobre el movimiento de la pobreza en el Santiago neoliberal. Elaboración propia, 2016.

\section{La eficacia de la suspensión legal}

La casa sólida se ha vuelto un bien ligero. Una mujer se inclina sobre el retrete que fue desinstalado de su domicilio. No está claro el destino del artefacto, pero es el foco central sobre el que dos personas impulsan un movimiento. El hombre se apoya sobre materiales sueltos (De Arteagabeitía, 1979:293), que evidencian el proceso de desensamble. Le da la espalda al observador; su objetivo es retomar el control del retrete que está alejado de su propósito funcional. Delante de la pareja se presentan otros objetos desarmados y arrumbados hacia la esquina izquierda. Una escalera insinúa su participación en el proceso de desarme. Está fuera de foco. El retrete es un símbolo que refiere al objeto transportado. El observador queda prendido de él como si estuviese transmitiendo señas.

Era el año 1977. Emilio Cartagena, dirigente de la junta de vecinos de La Nueva Matucana (Quinta Normal), informó que la población sería parte de las políticas estatales de erradicación de la pobreza (De Arteagabeitía, 1979:293294).. Así comenzó la presión militar y económica (la empresa Indus Lever negociaba con la municipalidad por el terreno) ${ }^{\mathbf{2}}$. Las casas poseían títulos de propiedad y los vecinos no estaban seguros de participar en la reasignación de viviendas (Délano, 1981:27). Aquí se evidenció la efectividad del estado de excepción ${ }^{2}$. Erradicar la pobreza se tradujo en trasladar a la clase baja. La municipalidad dificultó los trámites de compra-venta: desaparecieron papeles, las tasaciones se hicieron bajo el valor real y las personas fueron presionadas con engaños para firmar las ventas.

Los vecinos mantuvieron reuniones en un intento por resistir el traslado. Pero las casas, de cualquier manera, terminaron por desarmarse. Los titulares y entrevistas de la prensa de oposición (Délano, 1981:27) evidenciaron el importante rol de la unidad vecinal y de los atropellos le- gales que se habían sufrido. "O firmamos todos o ninguno" (Délano, 1981:27). "No se nos tomó el parecer" (De Arteagabeitía, 1979:293). La población fue sometida a la política del traslado a través de la omisión legal. La solución consistió en una doble firma: se recibió el dinero por el total de las propiedades pero, a la vez, se comprometieron a pagar un domicilio asignado a ciegas que terminaría costando un $70 \%$ más que su tasación real (Délano, 1981:27).

Las casas se demolieron. Nadie pudo oponerse a la operación de la que los hicieron parte. La prensa oficial sólo registró la población cuando quedaban los escombros. La pareja, sin embargo, cargó el retrete fuera de su domicilio. Nadie asegura que este haya vuelto a servir su propósito en la nueva propiedad. Su desplazamiento se mantiene en el plano de un artefacto inútil.

\section{La especulación disfrazada}

El vano de una ventana cumple el rol de marco. Dentro del bloque deshabitado, las ventanas inevitablemente dirigen la mirada: la construcción nueva está invadiendo el terreno. Las líneas negras atraviesan el fondo como una cruz. Un patio de tierra se extiende hasta un bloque vecino. En el plano medio, un poste de luz yace estropeado. Más allá, el bloque se vuelve a enmarcar, ahora dentro de un edificio que se posiciona sobre él. Algo va a pasar. Sobre aquellos cientos de vidrios, la mirada vuelve al marco original. Los cristales sucios y rotos de nuestra ventana quedan expuestos por encima del muro cortina. La opacidad del primer plano hace desaparecer el interior. El edificio nuevo se mantiene estable sobre el abandono.

Eduardo Frei Montalva inició el proyecto Renovación Parque San Luis con el objetivo crear un conjunto habitacional con una mayor diversidad de clases. Las Condes atravesaba un proceso de elitización, por lo que el proyecto no fue bien recibido. Los pobladores Ilegaron paulatina-

${ }^{1}$ Esta fue la opción elegida para proceder. Los vecinos fueron presionados a firmar los documentos de venta de las propiedades. ${ }^{\mathbf{2}}$ Entendido por Agamben como "[...] la apertura en la aplicación y la norma muestran su separación [...]" (Agamben, 2003:83). 

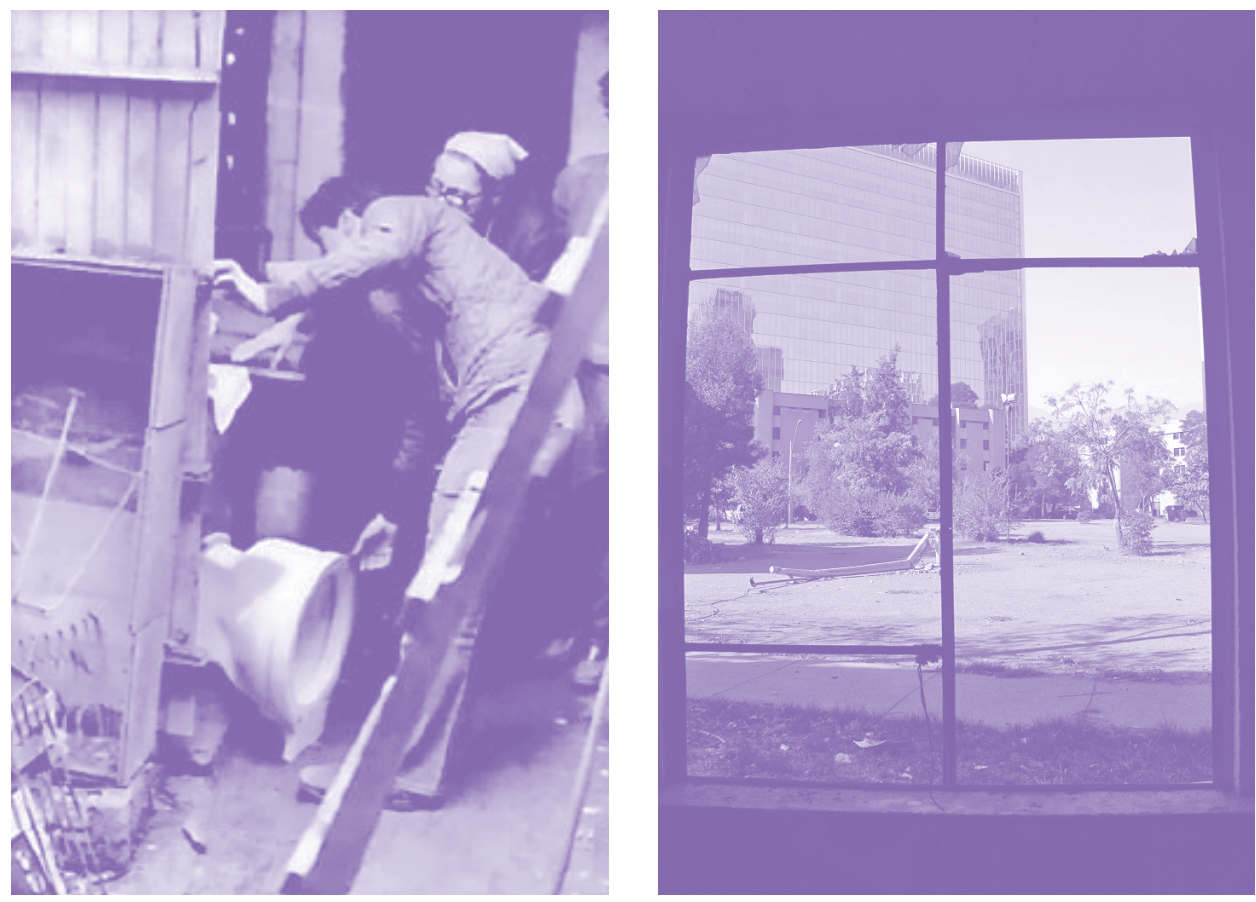

FIGURA 2 [IZQUIERDA].

Fotografía tomada en el periodo de desalojo de la Nueva Matucana. Fuente: Fondo de la Vicaría de la solidaridad, 1975-1979.

FIGURA 3 [DERECHA]. Fotografía tomada desde el Bloque 18 en Villa San Luis. Fuente: Toro, 2014.

mente en 1973. Los bloques terminados fueron habitados atendiendo a la necesidad (Toro, 2014). Sin embargo, no podían entregarse títulos de propiedad hasta que la obra estuviera terminada. Esto no alcanzó a ocurrir. Durante el golpe de Estado, antiguos funcionarios de CORHABIT y del MINvu intentaron regularizar la situación. Títulos de dominio provisorios fueron firmados y entregados (Toro, 2014). Pero en un estado de excepción un papel puede romperse fácilmente.

En 1975, en medio de la Operación Confraternidad I, las autoridades enviaron una carta notificando el desalojo y el traslado de la ocupación ilegal ${ }^{3}$. Pero no sólo hay que notar estos vacíos legales: el valor de suelo de la comuna subió un 400 \% desde 1983 a 1993 (Edwards, Hurtubia y Wagner, 1995:9) y este era un complejo habitacional destinado a la diversificación de clases, ¿por qué mantenerlo si podía perjudicar esta cifra? Desde 1976 hasta 1989 el ejército desalojó gran parte del complejo y consiguió adjudicarse su propiedad a través de Bienes Nacionales, lo que les permitió venderlo por partes durante la democracia (Díaz, 2015), mientras los pobladores restantes fueron declarados propietarios (Toro, 2014).

Los vidrios rotos continúan mirando el complejo de oficinas de Nueva Las Condes, ubicación ideal para los centros de negocios de Santiago. Los pobladores fueron convencidos de vender para completar este complejo de negocios. Los bloques se convirtieron en fantasmas. Las inmobiliarias llegaron a ofrecer $\$ 490$ millones por un sólo departamento del complejo (Toro, 2014). La especulación estaba disfrazada; marcó y sigue marcando la historia de esta villa modelo. Los nuevos edificios invadieron el conjunto y sus pobladores fueron una más de las diásporas de la época. Sólo queda la villa fantasma que se mira a sí misma a través de unas ventanas rotas.

\section{Una isla para los erradicados}

El peso de la línea equivale a la huella que deja un neumático. La ciudad representada en direcciones. Origen y destino están tensionándose y cambiando. El movimiento continuo de la ciudad nos deja este fragmento como una instantánea. Un momento que se toma en el avance y se suelta sin que decline. Cientos de miles de personas arrastradas en diáspora. Los barrios desarmados no pueden trasladarse. Se golpeó la puerta por la noche. En la mañana, el sitio es distinto, ajeno, obligatoriamente propio. La Pintana colapsa y se cierra a Santiago. Los camiones que llegan no volverán. La ciudad cinética es testigo de sí misma. El límite de la nueva comuna es como un cerco donde todos son iguales. El límite, la frontera, la inauguración de una ciudad distinta.

En el año 1981 se cambiaron los límites de la ciudad y se reacomodó la configuración de las comunas. La Pintana resultó de un conjunto de terrenos agrícolas que antes habían pertenecido a La Granja. Ya durante la dictadura, esta comuna fue convertida en el lugar de recepción para las personas erradicadas que no pudieron hacerse cargo de las

${ }^{3}$ Carta de Carlos Granifo Harms, Ministro de Vivienda y Urbanismo, con destino a Pdte. Junta de Vecinos de San Luis, 29 de diciembre, 1975. 
cuotas de pago de las primeras operaciones y de aquellos que habían estado en campamentos de emergencia y viviendas transitorias ${ }^{4}$. Los lugares cuyo valor en infraestructura y suelo eran mayores fueron 'limpiados' de campamentos y poblaciones indeseables. Así, la cantidad de erradicados alcanzó el 43,62 \% del total de la población de la comuna en 1984 (Morales y Rojas, 1986:82).

Los puntos, las flechas y las tablas muestran un entramado de acciones que acusan una línea de acción superior. El apunte se fija entonces en el líder de la dictadura. No es casual que Augusto Pinochet fuera geógrafo y que los planeamientos sobre la ciudad obedecieran a un movimiento centrífugo. La geopolítica pinochetista tenía como idea base la homogeneización de la población y el orden por valores de suelo y potencial económico en los distintos sectores de Santiago (Palacios y Leyton, 2012). Es por ello que no sorprende la creación de una comuna entera para la recepción de personas en igualdad de condiciones que estuvieron y, en algunos casos, están sumidas en la pobreza (Ortega, 2014:243).

Las tierras pasaron a urbanizarse a la fuerza. Los procedimientos realizados fuera de la legalidad, el interés depositado en los valores de suelo y las ideas de urbanización de la geopolítica configuraron el Santiago de hoy. El antiguo poblador se convirtió en propietario ${ }^{5}$. Al mismo tiempo, estas operaciones ampliaron el suelo potencialmente apto para especulación. La Pintana se habitó de erradicados y esto la destinó a ser el lugar de llegada de la pobreza, estableciendo un estigma sobre la comuna y sus habitantes. Los futuros planes de vivienda social retomaron estas lógicas de origen y destino. La isla se instaló como la imagen de estas zonas donde sólo se hacen perpetuos los procedimientos de segregación de quienes están en situación de vulnerabilidad (Ortega, 2014:261)..

\section{Conclusiones}

A través de los casos estudiados se pudo identificar algunos mecanismos utilizados en dictadura para la erradicación de la pobreza, teniendo el mecanismo económico cierta prevalencia por sobre los otros dos. Paralelo a esto, se ha manifestado la dificultad de entender estos mecanismos por sí solos y fue necesario cruzarlos para comprender su real complejidad. Su existencia reafirma la idea de que la erradicación de la pobreza no era el objetivo de las operaciones, pues ellas no la eliminaron, sólo la trasladaron de un punto central de la ciudad a uno más escondido, invisible. Además, junto a los objetivos de terminar con la pobreza, se situaron los objetivos socio-espaciales y económicos que homogeneizaron las comunas del Gran Santiago y abrieron nuevos mercados inmobiliarios en la ciudad.

Finalmente, el discurso construido en torno a las Operaciones Confraternidad sería el reflejo de la implementación de un sistema político, social y económico mayor. Pues el uso de la omisión por parte de las autoridades, a la vez de una propaganda que favorecía el discurso del fin de la pobreza, tuvo consecuencias mayores para los habitantes (sobre todo en el caso de La Pintana) y para la ciudad al dejar su desarrollo sujeto a las condiciones del mercado.

Entre todas estas dinámicas, el objeto fue un buen punto de partida para situar la narrativa de los casos estudiados. Sin embargo, la presencia de las demás fuentes y pruebas ha sido necesaria para entender los casos en sus diferencias y en sus puntos en común. El panorama que se intentó retratar durante el estudio podría construirse en la relación de estas fuentes como si se tratase de un cuadro histórico.

\section{REFERENCIAS BIBLIOGRÁFICAS}

Agamben, G. Estado de excepción. Homo sacer, II, I. Buenos Aires: Adriana Hidalgo Editora, 2003

De Arteagabeitía, R. "No se nos tomó el parecer". Mensaje 279 (1979).

Délano, M. Revista Hoy 201 (1981)

Díaz, J. "Cómo el ejército vendió el último terreno de Villa San Luis". The Clinic. Versión on-line. 2015. Revisado el 16/11/2016 <http://www.theclinic.cl/2015/06/o9/ como-el-ejercito-vendio-el-ultimo-terreno-de-villa-san-luis/>

Edwards, G.; Hurtubia, J.; y Wagner G. "El suelo urbano y el mercado de activos". Documento de trabajo N 177. Economía UC. 1995.

El Cabildo. "Sala de estar de día, dormitorio de noche". 1985.

Gurovich, A. "La Pintana, Una ciudad interminable". Revista de Urbanismo 1 (1999). Morales, E. y Rojas, S. "Relocalización socio-espacial de la pobreza. Política estatal y presión popular, 1979-1985". FLACSO, 280 (1986)

Ortega, T. "Criminalización y concentración de la pobreza en barrios segregados. Síntomas de guetización en La Pintana, Santiago de Chile". EURE. Vol. 40, 120 (2014).

Palacios, C. y Leyton, C. "Las olvidadas erradicaciones de la dictadura". El Ciudadano. Versión on-line. 2012. Revisado el 16/11/2016 desde <http://www.elciudadano. $\mathrm{cl} / 2012 / 12 / 17 / 61685 /$ las-olvidadas-erradicaciones-de-la-dictadura/>

Stackhouse, J. "The state of Housing, The Business of the State: The consequences of housing and urban development policies developed by the entrepreneurial state in Chile (1973-1989"). Tesis para optar al título de Doctor. Syracuse University, USA, 2007

Toro, I. "La caída del último bastión de Allende”. The Clinic. Versión on-line. 2014 Revisado el 18/11/16 en <http://www.theclinic.cl/2014/05/19/villa-san-luis-lacaidadel-ultimo-bastion-de-allende-en-las-condes/>

\footnotetext{
4 "Erradicaciones 1, Derecho a un hogar". Revista Solidaridad 26 (1979): 17. 5 "De ex pobladores a flamantes propietarios". La Tercera, 11 de septiembre de $1977,1$.
} 


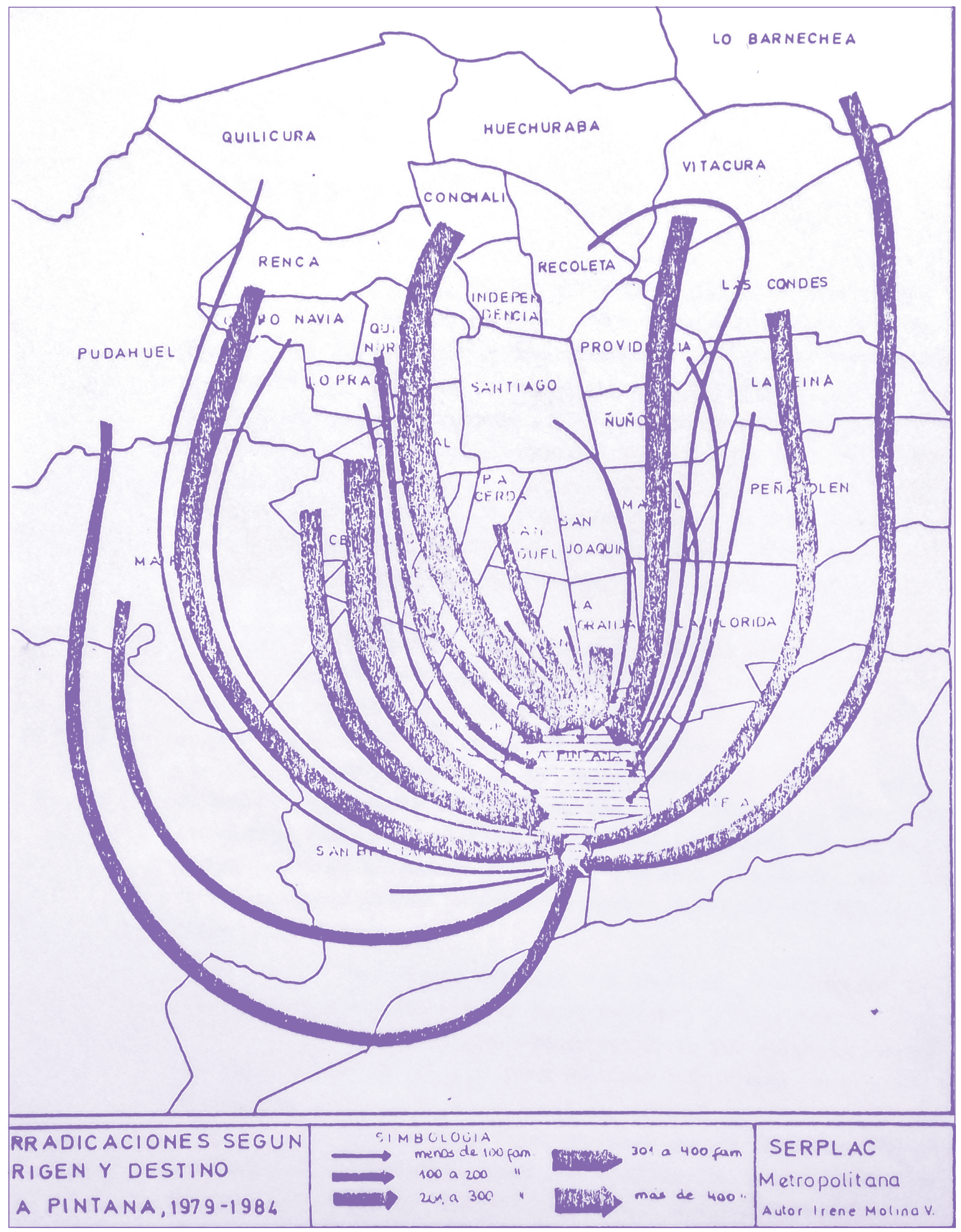

FIGURA 4.

Plano de erradicaciones con destino a La Pintana entre 1979 y 1984. Fuente: Molina, I. Erradicaciones según origen y destino. SERPLAC, I. Municipalidad de La Pintana. 